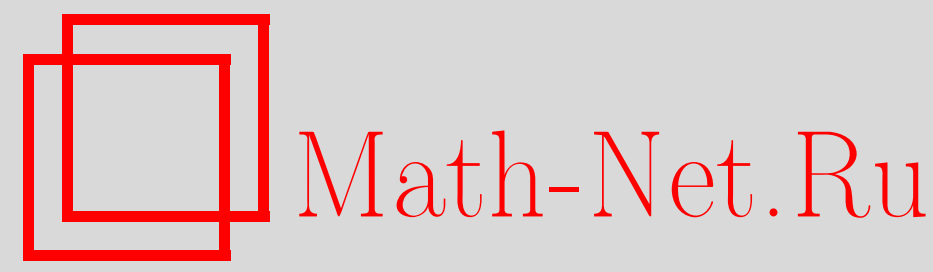

Ф. М. Мухамедов, О разложении квантовых квадратичных стохастических процессов на слойно-марковские процессы, определенные на алгебрах фон Неймана, Изв. РАН. Сер. матем., 2004, том 68, выпуск 5, 171-188

DOI: https://doi.org/10.4213/im506

Использование Общероссийского математического портала Math-Net.Ru подразумевает, что вы прочитали и согласны с пользовательским соглашением

http://www. mathnet.ru/rus/agreement

Параметры загрузки:

IP: 54.209 .52 .79

26 апреля 2023 г., 16:53:44 


\title{
О разложении квантовых квадратичных стохастических процессов на слойно-марковские процессы, определенные на алгебрах фон Неймана
}

\begin{abstract}
Приведено разложение квантового квадратичного стохастического процесса (к.к.с.п.) на так называемый слойно-марковский процесс, и, обратно, если существует такое разложение, то единственным образом можно восстановить квантовый квадратичный стохастический процесс. В качестве приложения дан критерий выполнения эргодического принципа для к.к.с.п. через полученное разложение. При использовании этого результата доказано, что к.к.с.п. удовлетворяет эргодическому принципу тогда и только тогда, когда ассоциированный марковский процесс удовлетворяет этому принципу. Далее с помощью полученного разложения введено новое понятие сопряженности двух к.к.с.п. и изучена связь этого понятия с эргодическим принципом.

Библиография: 21 наименование.
\end{abstract}

\section{§1. Введение}

Известно, что теория марковских процессов является бурно развивающимся направлением и имеет многочисленные применения в разных областях математики и физики. Однако сушествуют физические системы, которые не описываются марковскими процессами, например квадратичные стохастические процессы (см. [1]).

Квадратичные стохастические процессы связаны с понятием квадратичного стохастического оператора, которое впервые было сформулировано в работе [2]. Далее в работе [3] была поставлена задача изучения поведения траекторий квадратичных стохастических операторов. В [4]-[9] изучались предельное поведение и эргодические свойства траекторий квадратичных операторов.

В работах [10]-[12] определен квадратичный стохастический процесс, который естественным образом возникает при изучении некоторых моделей со взаимодействиями. Но они не охватывают случаи квантовых систем, так что возникает задача определения квантовых квадратичных процессов. В работах [13], [14] были введены квантовые квадратичные стохастические процессы, определенные на алгебрах фон Неймана, которые, в частности, включают квадратичные стохастические процессы. В работах [15], [16] рассматривались некоторые эргодические свойства квантовых квадратичных стохастических процессов (к.к.с.п.).

Задачей теории разложения, или декомпозиции, является выражение объектов сложной структуры через более простые компоненты. Не существует общего правила, объясняющего, что подразумевается под термином "более простые компоненты”, вопрос этот каждый раз решается конкретно. При изучении эргодических 
свойств к.к.с.п. возникают проблемы, связанные с тем, что этот процесс отображает алгебру фон Неймана $\mathscr{M}$ не на саму алгебру, а на ее тензорный квадрат $\mathscr{M} \otimes \mathscr{M}$. Это приводит к следующей проблеме: нельзя ли к.к.с.п. разложить на какие-то процессы, составленные из линейных операторов таких, что они отображают алгебру фон Неймана в себя?

Настоящая работа является продолжением работы [17], здесь дано разложение к.к.с.п. на так называемые слойно-марковские процессы, и, обратно, если имеется такое разложение, то доказывается, что единственным образом можно восстановить к.к.с.п. В качестве приложения приведен критерий, устанавливающий эргодический принцип для к.к.с.п. через полученное разложение. Используя этот результат, докажем, что к.к.с.п. удовлетворяет эргодическому принщипу тогда и только тогда, когда ассоциированный марковский процесс удовлетворяет этому принципу. Кроме того, с помошью полученного разложения к.к.с.п. определим понятие сопряженности двух к.к.с.п. и исследуем связь этого понятия с эргодическим принципом.

Заметим, что часть результатов настоящей работы была анонсирована в работе [18].

\section{§ 2. Предварительные сведения и определения}

Пусть $B(H)$ - алгебра всех линейных ограниченных операторов на комплексном гильбертовом пространстве $H$. Слабо замкнутая *-подалгебра $\mathscr{M}$ в $B(H)$ называется алгеброй фон Неймана, если она содержит единичный оператор 1. Элемент $x \in \mathscr{M}$ называется положительным, если существует элемент $y \in \mathscr{M}$ такой, что $x=y^{*} y$. Совокупность всех положительных элементов $\mathscr{M}$ обозначается как $\mathscr{M}_{+}$. Линейный функционал $\omega$ на $\mathscr{M}$ назьвается положительным, если $\omega(x) \geqslant 0$ для всех $x \in \mathscr{M}_{+}$. Совокупность всех положительных функционалов обозначим $\mathscr{M}_{+}^{*}$. Положительный функционал $\omega$ называется состоянием, если $\omega(\mathbf{1})=1$. Состояние $\omega$ нормально, если оно удовлетворяет равенству

$$
\omega\left(\sup _{\alpha} x_{\alpha}\right)=\sup _{\alpha} \omega\left(x_{\alpha}\right)
$$

для каждой равномерно ограниченной возрастающей сети $\left\{x_{\alpha}\right\}$ положительных элементов из $\mathscr{M}$. Совокупность всех ультраслабо непрерывных функционалов обозначим через $\mathscr{M}_{*}$. Обозначим $\mathscr{M}_{*,+}=\mathscr{M}_{*} \cap \mathscr{M}_{+}^{*}$.

Пусть $\mathscr{M}$ - алгебра фон Неймана, действующая на гильбертовом пространстве $H$. Тогда

$$
\mathscr{M} \odot \mathscr{M}=\left\{\sum_{i=1}^{n} x_{i} \otimes y_{i} \mid x_{i}, y_{i} \in \mathscr{M}, i=\overline{1, n}, n \in \mathbb{N}\right\}
$$

является *-подалгеброй во множестве всех операторов на $H \otimes H$. Слабое (операторное) замыкание $\mathscr{M} \otimes \mathscr{M}$ в $B(H \otimes H)$ обозначается через $\mathscr{M} \otimes \mathscr{M}$ и называется тензорным произведением алгебры $\mathscr{M}$ на себя. (Подробнее об алгебрах фон Неймана см., например, [19].) 
Пусть $\mathscr{M}$ - алгебра фон Неймана. Через $S$ и $S^{2}$ обозначим совокупности всех нормальных состояний на $\mathscr{M}$ и $\mathscr{M} \otimes \mathscr{M}$ соответственно. Пусть $\varphi \in S$ - состояние; определим оператор $E_{\varphi}: \mathscr{M} \otimes \mathscr{M} \rightarrow \mathscr{M}$ условного ожидания на элементах вида $a \otimes b, a, b \in \mathscr{M}$, следуюшим образом:

$$
E_{\varphi}(a \otimes b)=\varphi(a) b
$$

и далее продолжим по линейности и непрерьвности до $\mathscr{M} \otimes \mathscr{M}$. Ясно, что $E_{\varphi}$ является положительным (т. е. $E_{\varphi} x \geqslant 0$ при $x \geqslant 0$ ) и $E_{\varphi} \mathbf{1}_{\mathscr{M}}=\mathbf{1}_{\mathscr{M} \otimes \mathscr{M}}$, где $\mathbf{1}_{\mathscr{M}}$ и $1 \mathscr{M} \otimes \mathscr{M}$ - единицы алгебр $\mathscr{M}$ и $\mathscr{M} \otimes \mathscr{M}$ соответственно.

Пусть $\left\{P^{s, t}: \mathscr{M} \rightarrow \mathscr{M} \otimes \mathscr{M}, s, t \in \mathbb{R}_{+}, t-s>1\right\}$ - семейство линейных операторов и $U: \mathscr{M} \otimes \mathscr{M} \rightarrow \mathscr{M} \otimes \mathscr{M}$ - линейный оператор такой, что $U(x \otimes y)=y \otimes x$ для всех $x, y \in \mathscr{M}$.

ОПРЕДЕЛЕНИЕ 2.1. Будем говорить, что пара $\left(P^{s, t}, \omega_{0}\right)$, где $\omega_{0} \in S$ - начальное состояние, образует квантовый квадратичный стохастический прочесс (к.к.c.n.), если каждый оператор $P^{s, t}$ ультраслабо непрерывен и при этом выполнены следуюшие соотношения:

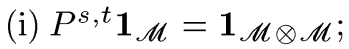

(ii) $P^{s, t}\left(\mathscr{M}_{+}\right) \subset(\mathscr{M} \otimes \mathscr{M})_{+}$;

(iii) $U P^{s, t} x=P^{s, t} x$ для любого $x \in \mathscr{M}$;

(iv) аналог уравнения Колмогорова-Чепмена: для произвольных $s, \tau, t \in \mathbb{R}_{+}$ таких, что $\tau-s \geqslant 1, t-\tau \geqslant 1$, выполнено какое-либо одно из двух следуюших равенств:

(iv) ${ }_{\mathrm{A}} P^{s, t} x=P^{s, \tau}\left(E_{\omega_{\tau}}\left(P^{\tau, t} x\right)\right), x \in \mathscr{M}$,

(iv) $)_{\mathrm{B}} P^{s, t} x=E_{\omega_{s}} P^{s, \tau} \otimes E_{\omega_{s}} P^{s, \tau}\left(P^{\tau, t} x\right), x \in \mathscr{M}$, где $\omega_{\tau}(x)=\omega_{0} \otimes \omega_{0}\left(P^{0, \tau} x\right), x \in \mathscr{M}$.

Если к.к.с.п. удовлетворяет равенству (iv) А или (iv) В, то будем говорить, что к.к.с.п. имеет тип (А) или тип (В) соответственно. Поскольку для физических, химических, биологических явлений необходимо некоторое время для реализации взаимодействия, то это время принимается за единицу времени (см. [12]). Поэтому $P^{s, t}$ определено при $t-s \geqslant 1$.

Примеры квантовых квадратичных стохастических процессов приведены в работе [18].

Заметим, что из условия (i) в силу [19, следствие 3.2 .6$]$ для любого $x \in \mathscr{M}$ имеет место неравенство

$$
\left\|P^{s, t} x\right\| \leqslant\|x\| \text {. }
$$

\section{§3. Разложение квантовых квадратичных стохастических процессов}

В этом параграфе дается разложение квантовых квадратичных стохастических процессов на слойно-марковские процессы.

Рассмотрим семейство отображений $\left\{H^{s, t}: \mathscr{M}_{*} \otimes_{\alpha_{0}^{*}} \mathscr{M}_{*} \rightarrow \mathscr{M}_{*}, s, t \in \mathbb{R}_{+}\right.$, $t-s \geqslant 1\}$, где $\alpha_{0}^{*}$ - дуальная норма к наименьшей $C^{*}$-кросс-норме $\alpha_{0}$ на $\mathscr{M} \otimes \mathscr{M}$, таких, что $H^{s, t}(\cdot \otimes \cdot)$ является билинейной функцией на $M_{*}$ и выполнены следующие соотношения: 
(i) $H^{s, t}\left(S^{2}\right) \subset S$;

(ii) $H^{s, t}(\varphi \otimes \psi)=H^{s, t}(\psi \otimes \varphi)$ для любых $\varphi, \psi \in S$;

(iii) для начального состояния $\omega_{0} \in S$ и чисел $s, \tau, t \in \mathbb{R}_{+}$таких, что $\tau-s \geqslant 1$, $t-\tau \geqslant 1$, выполнено какое-либо одно из двух следуюших равенств:

(iii) $H_{\mathrm{a}} H^{s, t}(\varphi)=H^{\tau, t}\left(H^{s, \tau}(\varphi) \otimes \omega_{\tau}\right), \varphi \in S^{2}$,

(iii) $)_{\mathrm{b}} H^{s, t}(\varphi \otimes \psi)=H^{\tau, t}\left(H^{s, \tau}\left(\omega_{s} \otimes \varphi\right) \otimes H^{s, \tau}\left(\omega_{s} \otimes \psi\right)\right), \varphi, \psi \in S$, где $\omega_{s}(x)=H^{0, s}\left(\omega_{0} \otimes \omega_{0}\right)(x)$.

Определенное таким образом семейство будем обозначать через $\left(H^{s, t}, \omega_{0}\right)$ и называть квадратичным процессом (к.n.) типа (А) или типа (В), в зависимости от того, которое из уравнений, (iii) $)_{\mathrm{a}}$ или (iii) $)_{\mathrm{b}}$, выполнено.

Заметим, что каждому к.к.с.п. $\left(P^{s, t}, \omega_{0}\right)$ можно сопоставить к.п. $\left(V^{s, t}, \omega_{0}\right)$ определенный следуюшим образом:

$$
V^{s, t}(\varphi)(a)=\varphi\left(P^{s, t} a\right), \quad \varphi \in \mathscr{M}_{*} \otimes_{\alpha_{0}^{*}} \mathscr{M}_{*}, \quad a \in \mathscr{M}
$$

Очевидно, что к.п. $\left(V^{s, t}, \omega_{0}\right)$ имеет тип (А) или тип (В), если к.к.с.п. $\left(P^{s, t}, \omega_{0}\right)$ имеет соответствуюший тип, так как из уравнения (iv) А (соответственно, (iv) В) следует справедливость уравнения (iii) a (соответственно, (iii) $)_{b}$.

Следующее предложение дает ответ на вопрос: можно ли по к.п. определить к.к.с.п. так, чтобы было верно равенство (3.1)?

ПРЕДЛОЖЕНИЕ 3.1. Каждый квадратичный прочесс $\left(H^{s, t}, \omega_{0}\right)$ определяет к.к.c.n. $\left(P^{s, t}, \omega_{0}\right)$ соответствующего типа такой, что справедливо равенство

$$
H^{s, t}(\varphi)(x)=\varphi\left(P^{s, t} x\right), \quad \varphi \in \mathscr{M}_{*} \otimes_{\alpha_{0}^{*}} \mathscr{M}_{*}, \quad x \in \mathscr{M} .
$$

ДоКАЗАТЕЛЬСТВо. Определим семейство отображений следующим образом:

$$
P^{s, t}:=\left(H^{s, t}\right)^{*}, \quad s, t \in \mathbb{R}_{+}, \quad t-s \geqslant 1 .
$$

В силу равенств $\left(\mathscr{M}_{*} \otimes_{\alpha_{0}^{*}} \mathscr{M}_{*}\right)^{*}=\mathscr{M} \otimes \mathscr{M},\left(\mathscr{M}_{*}\right)^{*}=\mathscr{M}$ (см. [20, определение $1.22 .10])$ ясно, что $P^{s, t}: \mathscr{M} \rightarrow \mathscr{M} \otimes \mathscr{M}$. Используя двойственную запись, равенство (3.3) теперь можно переписать следуюшим образом:

$$
\left\langle P^{s, t} x, \varphi\right\rangle=\left\langle x, H^{s, t} \varphi\right\rangle, \quad x \in \mathscr{M}, \quad \varphi \in \mathscr{M}_{*} \otimes_{\alpha_{0}^{*}} \mathscr{M}_{*} .
$$

Отсюда следует равенство (3.2).

Проверим, что $\left(P^{s, t}, \omega_{0}\right)$ является к.к.с.п. Ультраслабая непрерьвность каждого $P^{s, t}$ следует из условия (i) определения к.п. Пусть $\langle\mathbf{1}, \varphi\rangle \in \mathscr{M}, \varphi \in \mathscr{M}_{*}$; тогда

$$
\left\langle P^{s, t} \mathbf{1}, \psi\right\rangle=\left\langle\mathbf{1}, H^{s, t}(\psi)\right\rangle=H^{s, t}(\psi)(\mathbf{1})=1,
$$

где $\psi \in S^{2}$. Отсюда следует, что $P^{s, t} \mathbf{1}=\mathbf{1} \otimes \mathbf{1}$. Если $x \geqslant 0, x \in \mathscr{M}$, то $\langle x, \varphi\rangle \geqslant 0$ для всех $\varphi \in S$ и, следовательно, из условия (i) определения $H^{s, t}$ для любого $\psi \in S^{2}$ имеем

$$
\left\langle P^{s, t} x, \psi\right\rangle=\left\langle x, H^{s, t}(\psi)\right\rangle \geqslant 0,
$$


отсюда находим $P^{s, t} x \geqslant 0$. Из условия (ii) вытекает

$$
\left\langle P^{s, t} x, \varphi \otimes \psi\right\rangle=\left\langle P^{s, t} x, \psi \otimes \varphi\right\rangle
$$

что означает $P^{s, t} x \in\{x \in \mathscr{M} \otimes \mathscr{M} \mid U x=x\}$. Теперь рассмотрим отдельно два случая, когда к.п. $\left(H^{s, t}, \omega_{0}\right)$ имеет тип $(\mathrm{A})$ или тип $(\mathrm{B})$.

Пусть $\left(H^{s, t}, \omega_{0}\right)$ имеет тип $(\mathrm{A})$. Тогда, пользуясь уравнением (iii) $)_{\mathrm{a}}$, для чисел $s, \tau, t \in \mathbb{R}_{+}$таких, что $\tau-s \geqslant 1, t-\tau \geqslant 1$, имеем

$$
\begin{aligned}
\left\langle P^{s, t} x, \psi\right\rangle & =\left\langle x, H^{s, t}(\psi)\right\rangle=H^{s, t}(\psi)(x) \\
& =H^{\tau, t}\left(H^{s, \tau}(\psi) \otimes \omega_{\tau}\right)(x)=\left\langle P^{\tau, t} x, H^{s, \tau}(\psi) \otimes \omega_{\tau}\right\rangle \\
& =\left\langle E_{\omega_{\tau}}\left(P^{\tau, t} x\right), H^{s, \tau}(\psi)\right\rangle \\
& =\left\langle P^{s, \tau}\left(E_{\omega_{\tau}}\left(P^{\tau, t} x\right)\right), \psi\right\rangle, \quad x \in \mathscr{M}, \quad \psi \in S^{2} .
\end{aligned}
$$

Отсюда следует равенство

$$
P^{s, t} x=P^{s, \tau}\left(E_{\omega_{\tau}}\left(P^{\tau, t} x\right)\right), \quad x \in \mathscr{M}
$$

Итак, $\left(P^{s, t}, \omega_{0}\right)$ является к.к.с.п. типа (А).

Пусть теперь $\left(H^{s, t}, \omega_{0}\right)$ имеет тип (В). В этом случае мы используем уравнение (iii) $\mathrm{b}$. Для чисел $s, \tau, t \in \mathbb{R}_{+}$таких, как и выше, имеем

$$
\begin{aligned}
\left\langle P^{s, t} x, \varphi \otimes \psi\right\rangle & =\left\langle x, H^{s, t}(\varphi \otimes \psi)\right\rangle=H^{s, t}(\varphi \otimes \psi)(x) \\
& =H^{\tau, t}\left(H^{s, \tau}\left(\omega_{s} \otimes \varphi\right) \otimes H^{s, \tau}\left(\omega_{s} \otimes \psi\right)\right)(x) \\
& =\left\langle P^{\tau, t} x, H^{s, \tau}\left(\omega_{s} \otimes \varphi\right) \otimes H^{s, \tau}\left(\omega_{s} \otimes \psi\right)\right\rangle \\
& =\left\langle P^{s, \tau} \otimes P^{s, \tau}\left(P^{\tau, t} x\right),\left(\omega_{s} \otimes \varphi\right) \otimes\left(\omega_{s} \otimes \psi\right)\right\rangle \\
& =\left\langle E_{\omega_{s}} \otimes E_{\omega_{s}}\left(P^{s, \tau} \otimes P^{s, \tau}\right)\left(P^{\tau, t} x\right), \varphi \otimes \psi\right\rangle, \quad x \in \mathscr{M}, \quad \varphi, \psi \in S .
\end{aligned}
$$

Через $G$ обозначим линейную оболочку множества $\{\varphi \otimes \psi \mid \varphi, \psi \in S\}$. Из последних равенств находим

$$
\left(P^{s, t} x, \xi\right)=\left\langle E_{\omega_{s}} \otimes E_{\omega_{s}}\left(P^{s, \tau} \otimes P^{s, \tau}\right)\left(P^{\tau, t} x\right), \xi\right\rangle \quad \forall \xi \in G
$$

Поскольку $G$ сильно плотно в $S^{2}$, то можно получить равенство

$$
\left(P^{s, t} x, \varphi\right)=\left\langle E_{\omega_{s}} \otimes E_{\omega_{s}}\left(P^{s, \tau} \otimes P^{s, \tau}\right)\left(P^{\tau, t} x\right), \varphi\right\rangle \quad \forall \varphi \in S^{2}
$$

Отсюда следует равенство (iv) в в определении к.к.с.п. Таким образом, $\left(P^{s, t}, \omega_{0}\right)$ является к.к.с.п. типа (В). Предложение доказано.

Пусть $\mathscr{T}=\left\{T^{s, t}(\varphi): \mathscr{M} \rightarrow \mathscr{M} \mid s, t \in \mathbb{R}_{+}, t-s \geqslant 1, \varphi \in \mathscr{M}_{*}\right\}-$ семейство ультраслабо непрерьвных линейных отображений на алгебре фон Неймана $\mathscr{M}$. 
ОПРЕДЕЛЕНИЕ 3.1. Пара $\left(\mathscr{T}, \omega_{0}\right)$, где $\omega_{0} \in S$ - начальное состояние, назьвается слойно-марковским процессом (c.м.n.), если $T^{s, t}(\cdot)$ является линейной функцией на $\mathscr{M}_{*}$ при фиксированных значениях $s$ и $t$ и выполнены следующие условия:

(i) $T^{s, t}(\varphi) \mathbf{1}_{\mathscr{M}}=\mathbf{1}_{\mathscr{M}}$ при $\varphi \in S$;

(ii) $T^{s, t}(\varphi)\left(\mathscr{M}_{+}\right) \subset \mathscr{M}_{+}$при $\varphi \in \mathscr{M}_{*,+}$;

(iii) для начального состояния $\omega_{0} \in S$ и произвольных $s, \tau, t \in \mathbb{R}_{+}$таких, что $\tau-s \geqslant 1, t-\tau \geqslant 1$, выполнено какое-либо одно из двух следующих равенств:

(iii) $T^{s, t}(\varphi)=T^{s, \tau}(\varphi) T^{\tau, t}\left(\omega_{\tau}\right), \varphi \in S$,

(iii) ${ }_{\mathrm{B}} T^{s, t}(\varphi)=T^{s, \tau}\left(\omega_{s}\right) T^{\tau, t}\left(T_{*}^{s, \tau}\left(\omega_{s}\right) \varphi\right), \varphi \in S$, где $\omega_{\tau}(x)=\omega_{0}\left(T^{0, \tau}\left(\omega_{0}\right) x\right), x \in \mathscr{M}$, и $\left(T_{*}^{s, t}\left(\omega_{s}\right) \varphi\right)(x)=\varphi\left(T^{s, t}\left(\omega_{s}\right) x\right), x \in \mathscr{M}$.

Будем говорить, что с.м.п. $\left(\mathscr{T}, \omega_{0}\right)$ имеет тип $(\mathrm{A})$ или тип $(\mathrm{B})$ соответственно, в зависимости от того, которое из фундаментальных уравнений, (iii) А или (iii) В, имеет место.

ТЕОРемА 3.2. Каждый к.к.c.n. $\left(P^{s, t}, \omega_{0}\right)$ однозначно определяет с.м.n. $\left(\mathscr{T}_{p}, \omega_{0}\right)$ соответствующего типа следующим образом:

$$
T^{s, t}(\varphi) x=E_{\varphi}\left(P^{s, t} x\right), \quad \varphi \in \mathscr{M}_{*}, \quad x \in \mathscr{M} .
$$

При әтом для любых $\varphi, \psi \in S$ выполнено

$$
T_{*}^{s, t}(\varphi) \psi=T_{*}^{s, t}(\psi) \varphi, \quad\left\|T^{s, t}(\varphi)\right\| \leqslant 1 .
$$

Кроме того, имеет место равенство

$$
V^{s, t}(\varphi \otimes \psi)=T_{*}^{s, t}(\varphi) \psi \quad \forall \varphi, \psi \in S
$$

ДокАЗАТЕльСтво. Ясно, что из определения $T^{s, t}(\varphi)$ следует выполнение условий (i), (ii). Проверим справедливость равенств (iіi) определения 3.1. Пусть $s, \tau, t \in \mathbb{R}_{+}$такие, что $\tau-s \geqslant 1, t-\tau \geqslant 1$, и $\varphi \in S$. Из фундаментального уравнения (iv) А (соответственно, (iv) В ) получим

$$
\begin{aligned}
T^{s, t}(\varphi) x & =E_{\varphi}\left(P^{s, t} x\right)=E_{\varphi}\left(P^{s, \tau} E_{\omega_{\tau}}\left(P^{\tau, t} x\right)\right) \\
& =T^{s, \tau}(\varphi) T^{\tau, t}\left(\omega_{\tau}\right)(x), \quad x \in \mathscr{M}
\end{aligned}
$$

(соответственно,

$$
\begin{aligned}
T^{s, t}(\varphi) x & =E_{\varphi}\left(E_{\omega_{s}} \otimes E_{\omega_{s}}\left(P^{s, \tau} \otimes P^{s, \tau}\right)\left(P^{\tau, t} x\right)\right) \\
& =E_{\varphi}\left(\left(E_{\omega_{s}} P^{s, \tau} \otimes E_{\omega_{s}} P^{s, \tau}\right)\left(P^{\tau, t} x\right)\right) \\
& =T^{s, \tau}\left(\omega_{s}\right) \otimes T_{*}^{s, \tau}\left(\omega_{s}\right)(\varphi)\left(P^{\tau, t} x\right) \\
& =T^{s, \tau}\left(\omega_{s}\right) E_{T_{*}^{s, \tau}\left(\omega_{s}\right)(\varphi)}\left(P^{\tau, t} x\right) \\
& =T^{s, \tau}\left(\omega_{s}\right) T^{\tau, t}\left(T_{*}^{s, \tau}\left(\omega_{s}\right)\right)(x), \quad x \in \mathscr{M},
\end{aligned}
$$

где $\left.\omega_{s}(x)=\omega_{0} \otimes \omega_{0}\left(P^{0, s} x\right)=\omega_{0}\left(T^{0, s}\left(\omega_{0}\right) x\right)\right)$.

В силу условий (i)-(iii) определения 2.1 , очевидно, вьполнены соотношения (3.5) и (3.6). Теорема доказана.

Слойно-марковский процесс $\left(\mathscr{T}_{p}, \omega_{0}\right)$, заданный равенством $(3.4)$, будем называть разложсением к.к.с.п. на слойно-марковский процесс. Отсюда естественным образом возникает вопрос: можно ли через с.м.п. определить к.к.с.п. так, чтобы разложение этого к.к.с.п. на с.м.п. совпадало с данным первоначально?

Будем говорить, что с.м.п. ( $\left.\mathscr{T}, \omega_{0}\right)$ симметричен, если выполнено условие (3.5). 
ПРЕДЛОЖЕНИЕ 3.3. Каждый сим.метричный c.м.n. (T, $\left.\omega_{0}\right)$ определяет к.n. $\left(H^{s, t}, \omega_{0}\right)$ соответствующего типа так, что выполнено равенство

$$
H^{s, t}(\varphi \otimes \psi)=T_{*}^{s, t}(\varphi) \psi \quad \forall \varphi, \psi \in \mathscr{M}_{*} .
$$

ДокАЗАТЕЛЬСтво. Оператор $H^{s, t}$ определим на $G$ по линейности, где множество $G$ такое, как было определено при доказательстве предложения 3.1. В силу симметричности $\left(\mathscr{T}, \omega_{0}\right)$ для $f \in \mathscr{M}_{*}$ легко убедиться в справедливости следующего неравенства:

$$
\left\|T^{s, t}(f)\right\| \leqslant 2\|f\|_{1}
$$

Отсюда находим, что $H^{s, t}$ является непрерьвньм оператором на всюду плотном множестве $G$. Таким образом, его можно продолжить до $S^{2}$. Продолженный оператор обозначим также через $H^{s, t}$. Из определения $H^{s, t}$ легко следует выполнение

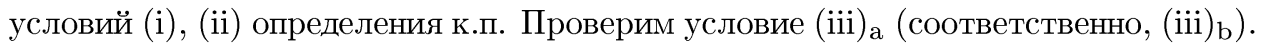
Для произвольных $s, \tau, t \in \mathbb{R}_{+}$таких, что $\tau-s \geqslant 1, t-\tau \geqslant 1$, имеем

$$
\begin{aligned}
H^{s, t}(\varphi \otimes \psi)(x) & =\left(T_{*}^{s, t}(\varphi) \psi\right)(x)=\psi\left(T^{s, t}(\varphi) x\right) \\
& =\psi\left(T^{s, \tau}(\varphi) T^{\tau, t}\left(\omega_{\tau}\right) x\right)=T_{*}^{\tau, t}\left(\omega_{\tau}\right) T_{*}^{s, \tau}(\varphi) \psi(x) \\
& =H^{\tau, t}\left(H^{s, \tau}(\varphi \otimes \psi) \otimes \omega_{\tau}\right)(x), \quad x \in \mathscr{M}, \quad \varphi, \psi \in S,
\end{aligned}
$$

где $\omega_{\tau}(x)=\omega_{0}\left(T^{0, \tau}\left(\omega_{0}\right) x\right)=H^{0, \tau}\left(\omega_{0} \otimes \omega_{0}\right)(x), x \in \mathscr{M}$ (соответственно,

$$
\begin{aligned}
H^{s, t}(\varphi \otimes \psi)(x) & =\psi\left(T^{s, t}(\varphi) x\right) \\
& =\psi\left(T^{s, \tau}\left(\omega_{s}\right) T^{\tau, t}\left(T_{*}^{s, \tau}\left(\omega_{s}\right) \varphi\right) x\right) \\
& =T_{*}^{\tau, t}\left(H^{s, \tau}\left(\omega_{s} \otimes \varphi\right)\right) T_{*}^{s, \tau}\left(\omega_{s}\right) \psi(x) \\
& =T_{*}^{\tau, t}\left(H^{s, \tau}\left(\omega_{s} \otimes \varphi\right)\right) H^{s, \tau}\left(\omega_{s} \otimes \psi\right)(x) \\
& \left.=H^{\tau, t}\left(H^{s, \tau}\left(\omega_{s} \otimes \psi\right) \otimes H^{s, \tau}\left(\omega_{s} \otimes \varphi\right)\right)(x), \quad x \in \mathscr{M}, \quad \varphi, \psi \in S\right) .
\end{aligned}
$$

Отсюда, повторяя рассуждения, проведенные при доказательстве предложения 3.1 , получим

$$
H^{s, t}(\varphi)=H^{\tau, t}\left(H^{s, \tau}(\varphi) \otimes \omega_{\tau}\right) \quad \forall \varphi \in S^{2} .
$$

Итак, $\left(H^{s, t}, \omega_{0}\right)$ является к.п. типа (А) (соответственно, типа (В)). Предложение доказано.

ТЕОРема 3.4. Каждый сим.метричный c.м.n. $\left(\mathscr{T}, \omega_{0}\right)$ определяет к.к.c.n. $\left(P^{s, t}, \omega_{0}\right)$ соответствующего типа так, ито имеет место равенство

$$
T^{s, t}(\varphi) x=E_{\varphi}\left(P^{s, t} x\right), \quad x \in \mathscr{M}, \quad \varphi \in \mathscr{M}_{*} .
$$


ДокАЗАТЕЛЬСтво. В силу предложения 3.3 с.м.п. $\left(\mathscr{T}, \omega_{0}\right)$ соответствует к.п. $\left(H^{s, t}, \omega_{0}\right)$ такой, что

$$
H^{s, t}(\varphi \otimes \psi)=T_{*}^{s, t}(\varphi) \psi \quad \forall \varphi, \psi \in \mathscr{M}_{*}
$$

Согласно предложению 3.1 этому к.п. соответствует к.к.с.п. $\left(P^{s, t}, \omega_{0}\right)$ такой, что

$$
H^{s, t}(\varphi \otimes \psi)(x)=\varphi \otimes \psi\left(P^{s, t} x\right), \quad x \in \mathscr{M}, \quad \varphi, \psi \in \mathscr{M}_{*} .
$$

Отсюда при фиксированном $\varphi \in \mathscr{M}_{*}$ находим

$$
T_{*}^{s, t}(\varphi) \psi(x)=\psi\left(E_{\varphi}\left(P^{s, t} x\right)\right), \quad x \in \mathscr{M}, \quad \forall \psi \in \mathscr{M}_{*} .
$$

В силу того, что $\mathscr{M}_{*}$ разделяет точки $\mathscr{M}$, получим равенство

$$
T^{s, t}(\varphi)(x)=E_{\varphi}\left(P^{s, t} x\right), \quad x \in \mathscr{M}, \quad \varphi \in \mathscr{M}_{*} .
$$

Теорема доказана.

Итак, из теорем 3.2 и 3.4 следует, что каждый к.к.с.п. $\left(P^{s, t}, \omega_{0}\right)$ однозначно разлагается на симметричный с.м.п. $\left(\mathscr{T}, \omega_{0}\right)$.

\section{§4. Связь слойно-марковского процесса с эргодическим принципом}

Пусть $\left(P^{s, t}, \omega_{0}\right)$ - к.к.с.п. на алгебре фон Неймана $\mathscr{M}$. Будем говорить, что к.к.с.п. $\left(P^{s, t}, \omega_{0}\right)$ удовлетворяет әргодическому приниипу, если для любых $\varphi, \psi \in S^{2}$ и $s \in \mathbb{R}_{+}$имеем

$$
\lim _{t \rightarrow \infty}\left\|V^{s, t} \varphi-V^{s, t} \psi\right\|_{1}=0
$$

где $\|\cdot\|_{1}-$ норма на $\mathscr{M}^{*}$ и $V^{s, t}-$ к.п., соответствующий $P^{s, t}$.

Отметим, что понятие эргодического принципа было введено впервые для марковского процесса в работах А.Н. Колмогорова, например [21], а для квадратичных процессов - в работе [11].

ТЕОРема 4.1. Пусть $\left(P^{s, t}, \omega_{0}\right)$ - к.к.c.n. на алгебре фон Неймана $\mathscr{M}$, и пусть $\left(\mathscr{T}, \omega_{0}\right)$ - его разложение на с.м.п. Тогда следующие условия эквивалентны:

(i) א.к.c.n. $\left(P^{s, t}, \omega_{0}\right)$ удовлетворяет эргодическому приниипу;

(ii) для любьх $\sigma, \varphi, \psi \in S u s \in \mathbb{R}_{+}$имеет место

$$
\lim _{t \rightarrow \infty}\left\|T_{*}^{s, t}(\sigma) \varphi-T_{*}^{s, t}(\sigma) \psi\right\|_{1}=0
$$

(iii) для любих $\sigma, \varphi, \psi \in \mathscr{R}$, где $\mathscr{R}-$ всюду плотное множество в $S$, и $s \in \mathbb{R}_{+}$ имеет место

$$
\lim _{t \rightarrow \infty}\left\|T_{*}^{s, t}(\sigma) \varphi-T_{*}^{s, t}(\sigma) \psi\right\|_{1}=0
$$


ДокАЗАТЕЛЬСТво. Импликации (i) $\Rightarrow($ ii) $\Rightarrow$ (iii) очевидны. Докажем импликацию (iii) $\Rightarrow$ (ii). Пусть $\sigma, \varphi, \psi \in S$. В силу плотности $\mathscr{R}$ для любого $\varepsilon>0$ сушествуют состояния $\eta, \xi, \zeta \in \mathscr{R}$ такие, что

$$
\|\sigma-\eta\|_{1}<\varepsilon, \quad\|\varphi-\xi\|_{1}<\varepsilon, \quad\|\psi-\zeta\|_{1}<\varepsilon .
$$

В силу неравенства (3.7) выполнено следующее неравенство:

$$
\left\|T_{*}^{s, t}(f) g\right\| \leqslant 2\|f\|_{1}\|g\|_{1}, \quad f, g \in \mathscr{M}_{*} .
$$

Отсюда имеем

$$
\left\|T_{*}^{s, t}(\eta) \xi-T_{*}^{s, t}(\sigma) \varphi\right\|_{1}<4 \varepsilon, \quad\left\|T_{*}^{s, t}(\eta) \zeta-T_{*}^{s, t}(\sigma) \psi\right\|_{1}<4 \varepsilon .
$$

В силу условия (iii) для состояний $\eta, \xi, \zeta \in \mathscr{R}$ и числа $s \in \mathbb{R}_{+}$сушествует $t_{0} \in \mathbb{R}_{+}$ такое, что

$$
\left\|T_{*}^{s, t}(\eta) \xi-T_{*}^{s, t}(\eta) \zeta\right\|_{1}<\varepsilon, \quad t \geqslant t_{0} .
$$

Таким образом, из соотношений (4.1)-(4.3) следует неравенство

$$
\left\|T_{*}^{s, t}(\sigma) \varphi-T_{*}^{s, t}(\sigma) \psi\right\|_{1}<9 \varepsilon,
$$

т. е. справедливо условие (ii).

Теперь докажем импликацию (ii) $\Rightarrow$ (i). Пусть $\psi_{i}, \psi_{1, i}, \varphi, \nu, i=\overline{1, p}$, принадлежат $S$, и пусть $\left\{\lambda_{i}\right\}_{i=1}^{p}$ - такие числа, что $\lambda_{i} \geqslant 0, \sum_{i=1}^{p} \lambda_{i}=1$. Тогда справедливо следующее тождество:

$$
\sum_{i=1}^{p} \lambda_{i} \psi_{i} \otimes \psi_{1, i}-\varphi \otimes \nu=\sum_{i=1}^{p} \lambda_{i} \psi_{i} \otimes\left(\psi_{1, i}-\nu\right)+\left(\sum_{i=1}^{p} \lambda_{i} \psi_{i}-\varphi\right) \otimes \nu .
$$

Обозначая $\psi=\sum_{i=1}^{p} \lambda_{i} \psi_{i}$, получим

$$
\sum_{i=1}^{p} \lambda_{i} \psi_{i} \otimes \psi_{1, i}-\varphi \otimes \nu=\sum_{i=1}^{p} \lambda_{i} \psi_{i} \otimes\left(\psi_{1, i}-\nu\right)+(\psi-\varphi) \otimes \nu
$$

Следовательно, пользуясь теоремой 3.2, имеем

$$
\begin{aligned}
\left\|V^{s, t}\left(\sum_{i=1}^{p} \lambda_{i} \psi_{i} \otimes \psi_{1, i}\right)-T_{*}^{s, t}(\varphi) \nu\right\|_{1} \leqslant & \sum_{i=1}^{p} \lambda_{i}\left\|T_{*}^{s, t}\left(\psi_{i}\right) \psi_{1, i}-T_{*}^{s, t}\left(\psi_{i}\right) \nu\right\|_{1} \\
& +\left\|T_{*}^{s, t}(\psi) \nu-T_{*}^{s, t}(\varphi) \nu\right\|_{1}
\end{aligned}
$$

В силу условия (ii) и симметричности с.м.п. $\mathscr{T}$ из (4.4) вытекает

$$
\left\|V^{s, t}\left(\sum_{i=1}^{p} \lambda_{i} \psi_{i} \otimes \psi_{1, i}\right)-T_{*}^{s, t}(\varphi) \nu\right\|_{1} \rightarrow 0, \quad t \rightarrow \infty .
$$


Таким образом, для произвольных состояний $\varphi_{j}, \varphi_{1, j}, \psi_{i}, \psi_{1, i}, \varphi, \psi \in S, i=\overline{1, p}$, $j=\overline{1, q}$, и чисел $\lambda_{i}, \mu_{j}$ таких, что $\sum_{i=1}^{p} \lambda_{i}=\sum_{j=1}^{q} \mu_{j}=1, \lambda_{i}, \mu_{j} \geqslant 0$, имеем

$$
\begin{aligned}
& \left\|V^{s, t}\left(\sum_{i=1}^{p} \lambda_{i} \psi_{i} \otimes \psi_{1, i}\right)-V^{s, t}\left(\sum_{j=1}^{q} \mu_{j} \varphi_{j} \otimes \varphi_{1, j}\right)\right\|_{1} \\
& \leqslant\left\|V^{s, t}\left(\sum_{i=1}^{p} \lambda_{i} \psi_{i} \otimes \psi_{1, i}\right)-T_{*}^{s, t}(\varphi) \psi\right\|_{1} \\
& \quad+\left\|V^{s, t}\left(\sum_{j=1}^{q} \mu_{j} \varphi_{j} \otimes \varphi_{1, j}\right)-T_{*}^{s, t}(\varphi) \psi\right\|_{1} \rightarrow 0, \quad t \rightarrow \infty .
\end{aligned}
$$

Напомним, что через $G$ мы обозначали выпуклую оболочку множества $\{\varphi \otimes \psi$ : $\varphi, \psi \in S\}$. Поскольку множество $G$ плотно в $S^{2}$ относительно $\sigma\left((\mathscr{M} \otimes \mathscr{M})_{*}, \mathscr{M} \otimes\right.$ $\mathscr{M})$-топологии, то для произвольного $\varepsilon>0$ и для любых $\varphi, \psi \in S^{2}$ найдутся состояния $\mu, \nu \in G$ такие, что

$$
\|\varphi-\mu\|_{1}<\frac{\varepsilon}{2}, \quad\|\psi-\nu\|_{1}<\frac{\varepsilon}{2}
$$

Для состояний $\mu$ и $\nu$ сушествует число $t_{0}=t_{0}(s, \mu, \nu)$ такое, что

$$
\left\|V^{s, t} \mu-V^{s, t} \nu\right\|_{1}<\frac{\varepsilon}{2} \quad \text { для всех } t \geqslant t_{0} .
$$

В силу последних неравенств и неравенства

$$
\left\|V^{s, t} \mu\right\|_{1} \leqslant\|\mu\|_{1} \quad \text { для всех } \mu \in(\mathscr{M} \otimes \mathscr{M})_{*},
$$

которое следует из соотношения (2.1), имеем

$$
\left\|V^{s, t} \varphi-V^{s, t} \psi\right\|_{1}<\varepsilon \text { при всех } t \geqslant t_{0} .
$$

Отсюда и следует требуемое. Теорема доказана.

Будем говорить, что с.м.п. ( $\left(\omega_{0}\right)$ удовлетворяет условию (Е), если сушествует число $\lambda \in[0,1)$ такое, что для любых $\varphi, \psi, \sigma \in S$ и $s \in \mathbb{R}_{+}$выполнено неравенство

$$
\left\|T_{*}^{s, t}(\sigma) \varphi-T_{*}^{s, t}(\sigma) \psi\right\|_{1} \leqslant \lambda\|\varphi-\psi\|_{1}
$$

хотя бы для одного $t=t(\varphi, \psi, \sigma, s) \in \mathbb{R}_{+}$.

Tеорема 4.2. Пусть $\left(P^{s, t}, \omega_{0}\right)$ - к.к.c.n. на алzебре фон Неймана $\mathscr{M}$, и пусть $\left(\mathscr{T}, \omega_{0}\right)$ - его разложение на с.м.п. Тогда следующие условия эквивалентны:

(i) к.к.c.n. $\left(P^{s, t}, \omega_{0}\right)$ удовлетворяет әргодическому принципу;

(ii) c.м.n. $\left(\mathscr{T}, \omega_{0}\right)$ удовлетворяет условию (E). 
ДокАЗАтельСтво. Рассмотрим импликацию (i) $\Rightarrow$ (ii). Пусть к.к.с.п. $\left(P^{s, t}, \omega_{0}\right)$ удовлетворяет эргодическому принципу. Тогда в силу [17, теорема 1$]$ существует число $\lambda \in[0,1)$ такое, что для любых $\xi, \eta \in S^{2}$ и $s \in \mathbb{R}_{+}$имеет место неравенство

$$
\left\|V^{s, t}(\xi)-V^{s, t}(\eta)\right\|_{1} \leqslant \lambda\|\xi-\eta\|_{1}
$$

хотя бы для одного $t=t(\varphi, \psi, \sigma, s) \in \mathbb{R}_{+}$. В качестве состояний $\xi$ и $\eta$ рассмотрим состояния $\sigma \otimes \varphi$ и $\sigma \otimes \psi$ соответственно, где $\sigma, \varphi, \psi \in S$. Тогда, пользуясь теоремой 3.2 , неравенство (4.5) примет следующий вид:

$$
\left\|T_{*}^{s, t}(\sigma) \varphi-T_{*}^{s, t}(\sigma) \psi\right\|_{1} \leqslant \lambda\|\sigma \otimes(\varphi-\psi)\|_{1}=\lambda\|\varphi-\psi\|_{1} .
$$

Таким образом, выполнено условие (Е).

Докажем импликацию (ii) $\Rightarrow$ (i). Рассмотрим два случая.

Случай (А). Пусть с.м.п. ( $\left.\mathscr{T}, \omega_{0}\right)$ имеет тип (А). Пусть состояния $\sigma, \varphi, \psi \in S$ и число $s \in \mathbb{R}_{+}$фиксированы. Тогда в силу условия (Е) существует число $t_{1} \in \mathbb{R}_{+}$ такое, что

$$
\left\|T_{*}^{s, t_{1}}(\sigma) \varphi-T_{*}^{s, t_{1}}(\sigma) \psi\right\|_{1} \leqslant \lambda\|\varphi-\psi\|_{1} .
$$

Для состояний $\omega_{t_{1}}, T_{*}^{s, t_{1}}(\sigma) \varphi, T_{*}^{s, t_{1}}(\sigma) \psi$ и числа $t_{1} \in \mathbb{R}_{+}$в силу условия (Е) существует число $t_{2} \in \mathbb{R}_{+}$такое, что справедливо неравенство

$$
\begin{gathered}
\left\|T_{*}^{t_{1}, t_{1}+t_{2}}\left(\omega_{t_{1}}\right)\left(T_{*}^{s, t_{1}}(\sigma) \varphi\right)-T_{*}^{t_{1}, t_{1}+t_{2}}\left(\omega_{t_{1}}\right)\left(T_{*}^{s, t_{1}}(\sigma) \psi\right)\right\|_{1} \\
\leqslant \lambda\left\|T_{*}^{s, t_{1}}(\sigma) \varphi-T_{*}^{s, t_{1}}(\sigma) \psi\right\|_{1} .
\end{gathered}
$$

В силу условия (iii) А определения 3.1 для всех $x \in \mathscr{M}$ имеем

$$
\begin{aligned}
T_{*}^{t_{1}, t_{1}+t_{2}}\left(\omega_{t_{1}}\right)\left(T_{*}^{s, t_{1}}(\sigma) \varphi\right)(x) & =T_{*}^{s, t_{1}}(\sigma) \varphi\left(T^{t_{1}, t_{1}+t_{2}}\left(\omega_{t_{1}}\right) x\right) \\
& =\varphi\left(T^{s, t_{1}}(\sigma) T^{t_{1}, t_{1}+t_{2}}\left(\omega_{t_{1}}\right) x\right) \\
& =\varphi\left(T^{s, t_{1}+t_{2}}(\sigma) x\right)=T_{*}^{s, t_{1}+t_{2}}(\sigma) \varphi(x) .
\end{aligned}
$$

Отсюда и из неравенств $(4.6),(4.7)$ находим, что

$$
\left\|T_{*}^{s, t_{1}+t_{2}}(\sigma) \varphi-T_{*}^{s, t_{1}+t_{2}}(\sigma) \psi\right\|_{1} \leqslant \lambda\left\|T_{*}^{s, t_{1}}(\sigma) \varphi-T_{*}^{s, t_{1}}(\sigma) \psi\right\|_{1} \leqslant \lambda^{2}\|\varphi-\psi\|_{1} .
$$

Предположим, что найдены такие числа $\left\{t_{i}\right\}_{i=1}^{m}$, что

$$
\left\|T_{*}^{s, K_{m}}(\sigma) \varphi-T_{*}^{s, K_{m}}(\sigma) \psi\right\|_{1} \leqslant \lambda^{m}\|\varphi-\psi\|_{1},
$$

где $K_{m}=\sum_{i=1}^{m} t_{i}$.

Покажем, что неравенство (4.8) справедливо для $m+1$. В силу условия теоремы для состояний $T_{*}^{s, K_{m}}(\sigma) \varphi, T_{*}^{s, K_{m}}(\sigma) \psi, \omega_{K_{m}}$ и числа $K_{m} \in \mathbb{R}_{+}$существует число $t_{m+1} \in \mathbb{R}_{+}$такое, что

$$
\begin{aligned}
& \left\|T_{*}^{K_{m}, K_{m}+t_{m+1}}\left(\omega_{K_{m}}\right)\left(T_{*}^{s, K_{m}}(\sigma) \varphi\right)-T_{*}^{K_{m}, K_{m}+t_{m+1}}\left(\omega_{K_{m}}\right)\left(T_{*}^{s, K_{m}}(\sigma) \psi\right)\right\|_{1} \\
& \leqslant \lambda\left\|T_{*}^{s, K_{m}}(\sigma) \varphi-T_{*}^{s, K_{m}}(\sigma) \psi\right\|_{1} .
\end{aligned}
$$


В силу условия (iii) А имеем

$$
\begin{aligned}
& T_{*}^{K_{m}, K_{m}+t_{m+1}}\left(\omega_{K_{m}}\right)\left(T_{*}^{s, K_{m}}(\sigma) \varphi\right)=T_{*}^{s, K_{m+1}}(\sigma) \varphi \\
& T_{*}^{K_{m}, K_{m}+t_{m+1}}\left(\omega_{K_{m}}\right)\left(T_{*}^{s, K_{m}}(\sigma) \psi\right)=T_{*}^{s, K_{m+1}}(\sigma) \psi .
\end{aligned}
$$

Отсюда и из неравенств (4.8), (4.9) получим

$$
\left\|T_{*}^{s, K_{m+1}}(\sigma) \varphi-T_{*}^{s, K_{m+1}}(\sigma) \psi\right\|_{1} \leqslant \lambda^{m+1}\|\varphi-\psi\|_{1},
$$

где $K_{m+1}=K_{m}+t_{m+1}$. Таким образом, по индукции находим, что неравенство (4.8) справедливо при всех $m \in \mathbb{N}$. Пусть $\varepsilon>0$ - произвольное число. Выберем $m \in \mathbb{N}$ такое, что $\lambda^{m}\|\varphi-\psi\|_{1}<\varepsilon$. Для $t \geqslant K_{m+1}$ имеем

$$
t=K_{m}+t_{m+1}+r, \quad 0 \leqslant r<t_{m+1} .
$$

Следовательно, получим

$$
\begin{aligned}
\left\|T_{*}^{s, t}(\sigma) \varphi-T_{*}^{s, t}(\sigma) \psi\right\|_{1} & =\left\|T_{*}^{K_{m}, t}\left(\omega_{K_{m}}\right)\left(T_{*}^{s, K_{m}}(\sigma) \varphi-T_{*}^{s, K_{m}}(\sigma) \psi\right)\right\|_{1} \\
& \leqslant\left\|T_{*}^{s, K_{m}}(\sigma) \varphi-T_{*}^{s, K_{m}}(\sigma) \psi\right\|_{1} \leqslant \lambda^{m}\|\varphi-\psi\|_{1}<\varepsilon .
\end{aligned}
$$

Теперь, пользуясь теоремой 4.1, получим, что к.к.с.п. $P^{s, t}$ типа (А) удовлетворяет эргодическому принщипу.

Случай (В). Предположим, что с.м.п. $\left(\mathscr{T}, \omega_{0}\right)$ имеет тип (В). Пусть состояния $\varphi, \psi \in S$ и число $s \in \mathbb{R}_{+}$фиксированы. В силу условия (Е) существует число $t_{1} \in \mathbb{R}_{+}$такое, что

$$
\left\|T_{*}^{s, t_{1}}\left(\omega_{s}\right) \varphi-T_{*}^{s, t_{1}}\left(\omega_{s}\right) \psi\right\|_{1} \leqslant \lambda\|\varphi-\psi\|_{1} .
$$

Для состояний $T_{*}^{s, t_{1}}\left(\omega_{s}\right) \omega_{s}, T_{*}^{s, t_{1}}\left(\omega_{s}\right) \varphi, T_{*}^{s, t_{1}}\left(\omega_{s}\right) \psi$ и числа $t_{1}$ в силу условия (Е) существует число $t_{2} \in \mathbb{R}_{+}$такое, что

$$
\begin{aligned}
& \left\|T_{*}^{t_{1}, t_{1}+t_{2}}\left(T_{*}^{s, t_{1}}\left(\omega_{s}\right) \omega_{s}\right) T_{*}^{s, t_{1}}\left(\omega_{s}\right) \varphi-T_{*}^{t_{1}, t_{1}+t_{2}}\left(T_{*}^{s, t_{1}}\left(\omega_{s}\right) \omega_{s}\right) T_{*}^{s, t_{1}}\left(\omega_{s}\right) \psi\right\| \\
& \leqslant \lambda\left\|T_{*}^{s, t_{1}}\left(\omega_{s}\right) \varphi-T_{*}^{s, t_{1}}\left(\omega_{s}\right) \psi\right\| .
\end{aligned}
$$

Заметим, что из условия (iii) В определения 3.1 для любых состояний $\varphi, \omega \in S$ и чисел $s, \tau, t \in \mathbb{R}_{+}$таких, что $\tau-s \geqslant 1, t-\tau \geqslant 1$, следует

$$
T_{*}^{s, t}(\varphi) \omega=T_{*}^{\tau, t}\left(T_{*}^{s, \tau}\left(\omega_{s}\right) \varphi\right) T_{*}^{s, t}\left(\omega_{s}\right) \omega .
$$

Из равенства (4.13) находим

$$
\begin{aligned}
& T_{*}^{t_{1}, t_{1}+t_{2}}\left(T_{*}^{s, t_{1}}\left(\omega_{s}\right) \omega_{s}\right) T_{*}^{s, t_{1}}\left(\omega_{s}\right) \varphi=T_{*}^{s, t_{1}+t_{2}}\left(\omega_{s}\right) \varphi \\
& T_{*}^{t_{1}, t_{1}+t_{2}}\left(T_{*}^{s, t_{1}}\left(\omega_{s}\right) \omega_{s}\right) T_{*}^{s, t_{1}}\left(\omega_{s}\right) \psi=T_{*}^{s, t_{1}+t_{2}}\left(\omega_{s}\right) \psi .
\end{aligned}
$$


Отсюда, подставляя полученное в (4.12) и учитывая неравенство (4.11), имеем

$$
\left\|T_{*}^{s, t_{1}+t_{2}}\left(\omega_{s}\right) \varphi-T_{*}^{s, t_{1}+t_{2}}\left(\omega_{s}\right) \psi\right\|_{1} \leqslant \lambda^{2}\|\varphi-\psi\|_{1}
$$

Аналогично, как и в случае (А), можно показать, что существуют такие числа $\left\{t_{i}\right\}_{i=1}^{m} \subset \mathbb{R}_{+}$, что

$$
\left\|T_{*}^{s, K_{m}}\left(\omega_{s}\right) \varphi-T_{*}^{s, K_{m}}\left(\omega_{s}\right) \psi\right\|_{1} \leqslant \lambda^{m}\|\varphi-\psi\|_{1}
$$

где $K_{m}=\sum_{i=1}^{m} t_{i}$.

Пусть $\varepsilon>0$ - произвольное число. Выберем $m \in \mathbb{N}$ так, чтобы $\lambda^{m}\|\varphi-\psi\|_{1}<\varepsilon$. Для числа $t \geqslant K_{m+1}$ имеем разложение (4.10). Следовательно, для состояния $\sigma \in S$, используя равенство (4.13) и неравенство (4.14), имеем

$$
\begin{aligned}
& \left\|T_{*}^{s, t}(\sigma) \varphi-T_{*}^{s, t}(\sigma) \psi\right\|_{1} \\
& \quad=\left\|T_{*}^{K_{m}, t}\left(T^{s, K_{m}}\left(\omega_{s}\right)_{*} \sigma\right) T^{s, K_{m}}\left(\omega_{s}\right)_{*} \varphi-T_{*}^{K_{m}, t}\left(T^{s, K_{m}}\left(\omega_{s}\right)_{*} \sigma\right) T^{s, K_{m}}\left(\omega_{s}\right)_{*} \psi\right\|_{1} \\
& \quad \leqslant\left\|T^{s, K_{m}}\left(\omega_{s}\right)_{*} \varphi-T^{s, K_{m}}\left(\omega_{s}\right)_{*} \psi\right\|_{1} \leqslant \lambda^{m}\|\varphi-\psi\|_{1}<\varepsilon .
\end{aligned}
$$

Отсюда, пользуясь теоремой 4.1, получим эргодический принщип. Теорема доказана.

ПРЕДЛОЖЕНИЕ 4.3. Пусть $P^{s, t}$ - к.к.c.n. на алгебре фон Неймана $\mathscr{M}$ $u\left(\mathscr{T}, \omega_{0}\right)$ - соответствующий ему с.м.n. Тогда для любых иисел $s, \tau, t \in \mathbb{R}_{+}$ таких, что $\tau-s \geqslant 1, t-\tau \geqslant 1$, для любого $x \in \mathscr{M}$ имеет место равенство

$$
T^{s, t}\left(\omega_{s}\right) x=T^{s, \tau}\left(\omega_{s}\right) T^{\tau, t}\left(\omega_{\tau}\right) x
$$

ДокАЗАТЕльСтво. Пусть к.к.с.п. $P^{s, t}$ имеет тип (А), тогда (4.15) непосредственно следует из равенства (iii) А определения 3.1. Пусть теперь к.к.с.п. имеет тип (В). Тогда для всех $x \in \mathscr{M}$ имеем

$$
\begin{aligned}
T_{*}^{s, \tau}\left(\omega_{s}\right) \omega_{s}(x) & =\omega_{s}\left(T^{s, \tau}\left(\omega_{s}\right) x\right)=T_{*}^{0, s}\left(\omega_{0}\right) \omega_{0}\left(T^{s, \tau}\left(\omega_{s}\right) x\right) \\
& =\omega_{0}\left(T^{0, s}\left(\omega_{0}\right) T^{s, \tau}\left(\omega_{s}\right) x\right) \\
& =\omega_{0}\left(T^{0, s}\left(\omega_{0}\right) T^{s, \tau}\left(T_{*}^{0, s}\left(\omega_{0}\right) \omega_{0}\right) x\right) \\
& =\omega_{0}\left(T^{0, \tau}\left(\omega_{0}\right) x\right)=\omega_{\tau}(x)
\end{aligned}
$$

здесь мы использовали равенство (iii)в определения 3.1.

Теперь переходим к доказательству равенства (4.15):

$$
T^{s, t}\left(\omega_{s}\right)=T^{s, \tau}\left(\omega_{s}\right) T^{\tau, t}\left(T^{s, \tau}\left(\omega_{s}\right) \omega_{s}\right)=T^{s, \tau}\left(\omega_{s}\right) T^{\tau, t}\left(\omega_{\tau}\right)
$$

Предложение доказано. 
СлЕДСТВИЕ 4.4. Пусть $P^{s, t}$ - к.к.c.n. на алгебре фон Неймана $\mathscr{M} u\left(\mathscr{T}, \omega_{0}\right)-$ его разложсение на с.м.n. Тогда следующие условия әквивалентны:

(i) $P^{s, t}$ удовлетворяет әргодическому принципу;

(ii) c.м.n. $\left(\mathscr{T}, \omega_{0}\right)$ удовлетворяет условию (E);

(iii) существует число $\lambda \in[0,1)$ такое, что для любих состояний $\varphi, \psi \in S$ и числа $s \in \mathbb{R}_{+}$справедливо неравенство

$$
\left\|T_{*}^{s, t}\left(\omega_{s}\right) \varphi-T_{*}^{s, t}\left(\omega_{s}\right) \psi\right\|_{1} \leqslant \lambda\|\varphi-\psi\|_{1}
$$

хотя биг для одного $t \in \mathbb{R}_{+}$.

ДокАЗАТЕльСтво. Импликации (i) $\Leftrightarrow$ (ii) следуют из теоремы 4.2, а импликация (ii) $\Rightarrow$ (iii) очевидна.

Докажем импликацию (iii) $\Rightarrow$ (i). Сначала предположим, что к.к.с.п. $P^{s, t}$ имеет тип (А). Пусть состояния $\varphi, \psi \in S$ и число $s \in \mathbb{R}_{+}$фиксированы. В силу условия (iii) существует число $t_{1} \in \mathbb{R}_{+}$такое, что

$$
\left\|T_{*}^{s, t_{1}}\left(\omega_{s}\right) \varphi-T_{*}^{s, t_{1}}\left(\sigma_{1}\right) \psi\right\|_{1} \leqslant \lambda\|\varphi-\psi\|_{1} .
$$

Для состояний $T_{*}^{s, t_{1}}\left(\omega_{s}\right) \varphi, T_{*}^{s, t_{1}}\left(\omega_{s}\right) \psi$ и числа $t_{1}$ в силу условия (iii) существует число $t_{2} \in \mathbb{R}_{+}$такое, что

$$
\begin{gathered}
\left\|T_{*}^{t_{1}, t_{1}+t_{2}}\left(\omega_{t_{1}}\right) T_{*}^{s, t_{1}}\left(\omega_{s}\right) \varphi-T_{*}^{t_{1}, t_{1}+t_{2}}\left(\omega_{t_{1}}\right) T_{*}^{s, t_{1}}\left(\omega_{s}\right) \psi\right\|_{1} \\
\leqslant \lambda\left\|T_{*}^{s, t}\left(\omega_{s}\right) \varphi-T_{*}^{s, t}\left(\omega_{s}\right) \psi\right\|_{1} \leqslant \lambda^{2}\|\varphi-\psi\|_{1}
\end{gathered}
$$

здесь мы использовали неравенство (4.16). В силу предложения 4.3 для любого состояния $\varphi \in S$ справедливо равенство

$$
T_{*}^{\tau, t}\left(\omega_{\tau}\right) T_{*}^{s, \tau}\left(\omega_{s}\right) \varphi=T^{s, t}\left(\omega_{s}\right) \varphi
$$

Пользуясь этим равенством, получим

$$
\left\|T_{*}^{s, t_{1}+t_{2}}\left(\omega_{s}\right) \varphi-T_{*}^{s, t_{1}+t_{2}}\left(\omega_{s}\right) \psi\right\|_{1} \leqslant \lambda^{2}\|\varphi-\psi\|_{1} .
$$

Аналогично, как и при доказательстве теоремы 4.2, получим

$$
\left\|T_{*}^{s, K_{m}}\left(\omega_{s}\right) \varphi-T_{*}^{s, K_{m}}\left(\omega_{s}\right) \psi\right\|_{1} \leqslant \lambda^{m}\|\varphi-\psi\|_{1},
$$

где $K_{m}=\sum_{i=1}^{m} t_{i}$. Отсюда следует, что

$$
\left\|T_{*}^{s, t}\left(\omega_{s}\right) \varphi-T_{*}^{s, t}\left(\omega_{s}\right) \psi\right\|_{1} \rightarrow 0, \quad t \rightarrow \infty
$$

Пусть $\sigma \in S$ - произвольное состояние; тогда для числа $\tau \geqslant s+1$, пользуясь равенством (4.17), имеем

$$
\begin{aligned}
\left\|T_{*}^{s, t}(\sigma) \varphi-T_{*}^{s, t}(\sigma) \psi\right\|_{1} & =\left\|T_{*}^{\tau, t}\left(\omega_{\tau}\right) T_{*}^{s, \tau}(\sigma) \varphi-T_{*}^{\tau, t}\left(\omega_{\tau}\right) T_{*}^{s, \tau}(\sigma) \psi\right\|_{1} \\
& =\left\|T_{*}^{\tau, t}\left(\omega_{\tau}\right) \tilde{\varphi}-T_{*}^{\tau, t}\left(\omega_{\tau}\right) \tilde{\psi}\right\|_{1} \rightarrow 0, \quad t \rightarrow \infty
\end{aligned}
$$


где $\tilde{\varphi}=T_{*}^{s, \tau} \varphi, \tilde{\psi}=T_{*}^{s, \tau} \psi$. Таким образом, из теоремы 4.1 следует выполнение эргодического принщипа.

Случай, когда к.к.с.п. $P^{s, t}$ имеет тип (В), доказывается аналогично. Следствие доказано.

Определим новый процесс $Q^{s, t}: \mathscr{M} \rightarrow \mathscr{M}$ следующим образом:

$$
Q^{s, t} x=T^{s, t}\left(\omega_{s}\right)(x), \quad x \in \mathscr{M} \text {. }
$$

Из предложения 4.3 следует, что процесс $Q^{s, t}$ является нестационарным марковским процессом. В работе [17] было отмечено, что если к.к.с.п. $P^{s, t}$ удовлетворяет эргодическому принципу, то марковский процесс $Q^{s, t}$ также удовлетворяет этому принципу. Но обратное утверждение не было известно. Из следствия 4.4 находим, что обратное утверждение также верно, т. е. имеет место следующая

TЕОрема 4.5. Пусть $P^{s, t}$ - к.к.c.n. на алгебра фон Неймана $\mathscr{M}$ и $Q^{s, t}$ соответствующий ему марковский процесс. Тогда следующие условия әквивалентны:

(i) к.к.c.n. $P^{s, t}$ удовлетворяет эргодическому приниипу;

(ii) марковский прочесс $Q^{s, t}$ удовлетворяет эргодическому принципу.

\section{$\S 5$. Сопряженные квантовые квадратичные стохастические процессы}

Полученное разложение к.к.с.п. позволяет нам определить понятие сопряженности двух к.к.с.п., определенных на алгебре фон Неймана, следующим образом.

Пусть $\left(P^{s, t}, \omega_{0}\right)$ и $\left(Q^{s, t}, \omega_{0}\right)$ - два к.к.с.п. на алгебре фон Неймана $\mathscr{M}$, и пусть $\left(\mathscr{T}_{P}, \omega_{0}\right)$ и $\left(\mathscr{T}_{Q}, \omega_{0}\right)$ - их разложения на с.м.п. соответственно. Будем говорить, что к.к.с.п. $\left(P^{s, t}, \omega_{0}\right)$ и $\left(Q^{s, t}, \omega_{0}\right)$ сопряжены, и обозначать это $P^{s, t} \sim Q^{s, t}$, если сушествует семейство автоморфизмов $\left\{\theta^{s, t}(\varphi): s, t \in \mathbb{R}_{+}, t-s \geqslant 1, \varphi \in \mathscr{M}_{*}\right\}$ такое, что для любых чисел $s, t \in \mathbb{R}_{+}, t-s \geqslant 1$, и функционала $\varphi \in \mathscr{M}_{*}$ имеет место равенство

$$
T_{P}^{s, t}(\varphi) \theta^{s, t}(\varphi)=T_{Q}^{s, t}(\varphi) .
$$

Ясно, что это отношение является отношением эквивалентности.

Приведем примеры сопряженных к.к.с.п.

Пример 5.1. Пусть $\mathscr{M}$ - алгебра фон Неймана и $Z: \mathscr{M} \rightarrow \mathscr{M}$ - ультраслабо непрерьвное линейное отображение такое, что:

(i) $Z \mathbf{1}=\mathbf{1}$;

(ii) $Z\left(\mathscr{M}_{+}\right) \subset \mathscr{M}_{+}$.

Такое отображение называется марковским оператором.

Пусть состояние $\omega_{0} \in S$ инвариантно относительно оператора $Z$, т. е. $\omega_{0}(Z x)=$ $\omega_{0}(x)$ для всех $x \in \mathscr{M}$. Тогда

$$
P_{Z}^{k, n} x=\frac{1}{2}\left(Z^{k, n} x \otimes \mathbf{1}+\mathbf{1} \otimes Z^{k, n} x\right),
$$

где

$$
Z^{k, n} x=\frac{1}{2^{n-k-1}}\left(Z^{n-k} x+\left(2^{n-k-1}-1\right) \omega_{0}(x) \mathbf{1}\right), \quad x \in \mathscr{M}
$$


$k \geqslant 0, n \in \mathbb{N}, k<n$, является к.к.с.п. с начальным состоянием $\omega_{0}$.

Пусть $\theta: \mathscr{M} \rightarrow \mathscr{M}$ - автоморфизм такой, что $\omega_{0}(\theta(x))=\omega_{0}(x)$ для всех $x \in \mathscr{M}$ и $\theta Z=Z \theta$. Положим $Z_{1}=Z \theta$. Тогда ясно, что $Z_{1}$ является марковским оператором с инвариантным состоянием $\omega_{0}$. Определим другой к.к.с.п., соответствующий $Z_{1}$, по указанному выше способу:

$$
P_{Z_{1}}^{k, n} x=\frac{1}{2}\left(Z_{1}^{k, n} x \otimes \mathbf{1}+\mathbf{1} \otimes Z_{1}^{k, n} x\right)
$$

где

$$
Z_{1}^{k, n} x=\frac{1}{2^{n-k-1}}\left(Z_{1}^{n-k} x+\left(2^{n-k-1}-1\right) \omega_{0}(x) \mathbf{1}\right), \quad x \in \mathscr{M}
$$

$k \geqslant 0, n \in \mathbb{N}, k<n$. Соответствующие им с.м.п. имеют следующий вид:

$$
\begin{aligned}
& T_{Z}^{k, n}(\varphi) x=\frac{1}{2}\left(\varphi\left(Z^{k, n} x\right) \mathbf{1}+\varphi(\mathbf{1}) Z^{k, n} x\right), \\
& T_{Z_{1}}^{k, n}(\varphi) x=\frac{1}{2}\left(\varphi\left(Z_{1}^{k, n} x\right) \mathbf{1}+\varphi(\mathbf{1}) Z_{1}^{n, k} x\right),
\end{aligned}
$$

где $\varphi \in \mathscr{M}_{*}, x \in \mathscr{M}$. Определим автоморфизмы $\theta^{k, n}(\varphi): \mathscr{M} \rightarrow \mathscr{M}$ следующим образом:

$$
\theta^{k, n}(\varphi)=\theta^{n-k} \quad \forall \varphi \in \mathscr{M}_{*}
$$

Тогда легко видеть, что равенство (5.1) вьполнено. Таким образом, к.к.с.п. $P_{Z}^{k, n}$ и $P_{Z_{1}}^{k, n}$ сопряжены.

ПримеР 5.2. Рассмотрим коммутативную конечномерную алгебру фон Неймана $l_{2}^{\infty}$. Известно [17], чтобы определить к.к.с.п. на рассматриваемой алгебре, достаточно задать коэффициенты $p_{i j, k}^{s, t}$, удовлетворяюшие условиям

$$
p_{i j, k}^{s, t} \geqslant 0, \quad p_{i j, k}^{s, t}=p_{j i, k}^{s, t}, \quad \sum_{k=1}^{n} p_{i j, k}^{s, t}=1
$$

где $i, j, k \in\{1,2\}$.

Пусть $x_{0}=(x, 1-x), x \in[0,1],-$ начальное состояние. Определим коэффициенты к.к.с.п. $P^{s, t}$ и $Q^{s, t}$ соответственно следующим образом:

$$
\begin{aligned}
p_{11,1}^{s, t} & =\frac{1}{2^{t-s-1}}\left(\left(2^{t-s-1}-1\right) x+1\right), \\
p_{12,1}^{s, t}=p_{21,1}^{s, t} & =\frac{1}{2^{t-s-1}}\left(\left(2^{t-s-1}-1\right) x+\frac{1}{2}\right), \\
p_{22,1}^{s, t} & =\frac{1}{2^{t-s-1}}\left(2^{t-s-1}-1\right) x, \\
p_{i j, 2}^{s, t} & =1-p_{i j, 1}^{s, t}, \quad i, j \in\{1,2\} \\
q_{11,1}^{s, t} & =\frac{1}{2^{t-s-1}}\left(2^{t-s-1}-1\right)(1-x), \\
q_{12,1}^{s, t}=q_{21,1}^{s, t} & =\frac{1}{2^{t-s-1}}\left(\left(2^{t-s-1}-1\right)(1-x)+\frac{1}{2}\right), \\
q_{22,1}^{s, t} & =\frac{1}{2^{t-s-1}}\left(\left(2^{t-s-1}-1\right)(1-x)+1\right), \\
q_{i j, 2}^{s, t} & =1-q_{i j, 1}^{s, t}, \quad i, j \in\{1,2\} .
\end{aligned}
$$


Тогда легко проверить, что они сопряжены; автоморфизмы $\theta^{s, t}(\varphi): l_{2}^{\infty} \rightarrow l_{2}^{\infty}$ определяются следующим образом:

$$
\theta^{s, t}(\varphi)\left(y_{1}, y_{2}\right)=\left(y_{2}, y_{1}\right) \quad \forall s, t \in \mathbb{R}_{+}, \quad t-s \geqslant 1
$$

где $y=\left(y_{1}, y_{2}\right) \in l_{2}^{\infty}, \varphi \in l_{2}^{1}\left(\left(l_{2}^{1}\right)^{*}=l_{2}^{\infty}\right)$.

Возникает вопрос: как соотносятся сопряженные к.к.с.п., если один из них удовлетворяет эргодическому принципу? Следующая теорема дает ответ на этот вопрос.

Teорема 5.1. Пусть $\left(P^{s, t}, \omega_{0}\right) u\left(Q^{s, t}, \omega_{0}\right)$ - coпряжснные к.к.c.n. на алгебре фон Неймана $\mathscr{M}$. Тогда $\left(P^{s, t}, \omega_{0}\right)$ удовлетворяет әргодическому принципу, если и только если $\left(Q^{s, t}, \omega_{0}\right)$ удовлетворяет әргодическому приниипу.

ДоКАЗАТЕЛЬСТВо. В силу симметричности достаточно доказать теорему в одну сторону. Пусть $\left(P^{s, t}, \omega_{0}\right)$ удовлетворяет эргодическому принципу. Тогда согласно теореме 4.1 для любых состояний $\varphi, \psi, \sigma \in S$ имеем

$$
\lim _{t \rightarrow \infty}\left\|T_{*, P}^{s, t}(\sigma) \varphi-T_{*, P}^{s, t}(\sigma) \psi\right\|_{1}=0
$$

Из равенства (5.1) находим

$$
\begin{aligned}
T_{*, Q}^{s, t}(\sigma) \varphi(x) & =\varphi\left(T_{Q}^{s, t}(\sigma) x\right) \\
& =\varphi\left(T_{P}^{s, t}(\sigma) \theta^{s, t}(\sigma) x\right)=\theta_{*}^{s, t}(\sigma) T_{*, P}^{s, t}(\sigma) \varphi(x), \quad \varphi \in S, \quad x \in \mathscr{M} .
\end{aligned}
$$

Следовательно, получим

$$
\begin{aligned}
\left\|T_{*, Q}^{s, t}(\sigma) \varphi-T_{*, Q}^{s, t}(\sigma) \psi\right\|_{1} & =\left\|\theta_{*}^{s, t}(\sigma)\left(T_{*, P}^{s, t}(\sigma) \varphi-T_{*, P}^{s, t}(\sigma) \psi\right)\right\|_{1} \\
& \leqslant\left\|T_{*, P}^{s, t}(\sigma) \varphi-T_{*, P}^{s, t}(\sigma) \psi\right\|_{1} .
\end{aligned}
$$

Отсюда вытекает

$$
\lim _{t \rightarrow \infty}\left\|T_{*, Q}^{s, t}(\sigma) \varphi-T_{*, Q}^{s, t}(\sigma) \psi\right\|_{1}=0 .
$$

Таким образом, используя теорему 4.1 , получим требуемое. Теорема доказана.

\section{Список литературы}

1. Больцман Л. Избранные труды. М.: Наука, 1984.

2. Бернштейн C.H. Решение одной математической проблемы, связанной с теорией наследственности // Уч. зап. НИ каф. Укр. отд. мат. 1924. № 1. С. 83-115.

3. Улам C. М. Нерешенные математические проблемы. М.: Наука, 1964.

4. Kesten H. Quadratic transformations: a model for population growth. I, II // Adv. Appl. Prob. 1970. № 2. P. 1-82; P. 179-228. 
5. Любич Ю. И. Основные понятия и теоремы эволюционной генетики свободных популяций // УМН. 1971. Т. 26. № 5. С. 51-116.

6. Сарымсаков Т.А., Ганиходжаев Р.Н. Эргодический принцип для квадратичных стохастических операторов // Изв. АН УзССР. Сер. физ.-мат. наук. 1979. №6. С. 34-39.

7. Валландер C.C. О предельном поведении последовательности итераций некоторых квадратичных преобразований // ДАН СССР. 1972. Т. 202. № 3. С. 515-517.

8. Максимов B. M. Кубические стохастические матрицы и их вероятностные интерпретации // Теор. вероятн. и ее примен. 1996. Т. 41. № 1. С. 89-106.

9. Любич Ю. И. Математические структуры в популяционной генетике. Киев: Наук. думка, 1983.

10. Сарьмсаков Т.А., Ганиходжаев Н.Н. Аналитические методы в теории квадратичных стохастических операторов // ДАН СССР. 1989. Т. 305. № 5. С. 1052-1056.

11. Сарьмсаков Т. А., Ганиходжаев Н. Н. Об эргодическом принципе для квадратичных процессов // ДАН СССР. 1991. Т. 316. №6. С. 1315-1319.

12. Sarymsakov T.A., Ganikhodzhaev N.N. Analytic methods in the theory of quadratic stochastic processes // J. Theor. Prob. 1990. V. 3. № 1. P. 51-70.

13. Ганиходжаев Н. Н., Мухамедов Ф. М. О квантовых квадратичных стохастических процессах // ДАН Респ. Узб. 1997. № 3. С. 13-16.

14. Ганиходжаев Н.H., Мухамедов Ф. М. О квантовых квадратичных стохастических процессах и некоторые эргодические теоремы для таких процессов // Узб. матем. журн. 1997. № 3. C. 8-20.

15. Ганиходжаев Н. Н., Мухамедов Ф. М. Эргодические свойства квантовых квадратичных стохастических процессов // УМН. 1998. Т. 53. №6. С. 243-244.

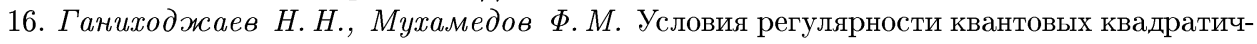
ных стохастических процессов // Докл. РАН. 1999. Т. 365. № 3. С. 301-303.

17. Ганиходжаев H. Н., Мухамедов $\Phi$. . Эргодические свойства квантовых квадратичных стохастических процессов // Изв. РАН. Сер. матем. 2000. Т. 65. № 5. С. 3-20.

18. Мухамедов Ф. М. О разложении квантовых квадратичных стохастических процессов // Докл. РАН. 2000. Т. 371. №2. С. 167-169.

19. Браттели У., Робинсон Д. Операторные алгебры и квантовая статистическая механика. М.: Мир, 1982.

20. Sakai S. $C^{*}$-algebras and $W^{*}$-algebras. N.Y.: Springer, 1971.

21. Колмогоров А.Н. Об аналитических методах в теории вероятностей // УМН. 1938. № 5. C. $5-51$. 